Western University

Scholarship@Western

Biology Publications

Biology Department

$3-1-2013$

\title{
Ontogenetic variation in cold tolerance plasticity in Drosophila: is the Bogert effect bogus?
}

Katherine A Mitchell

Brent J Sinclair

Western University, bsincla7@uwo.ca

John S Terblanche

Follow this and additional works at: https://ir.lib.uwo.ca/biologypub

Part of the Biology Commons

Citation of this paper:

Mitchell, Katherine A; Sinclair, Brent J; and Terblanche, John S, "Ontogenetic variation in cold tolerance plasticity in Drosophila: is the Bogert effect bogus?" (2013). Biology Publications. 77.

https://ir.lib.uwo.ca/biologypub/77 


\section{Naturwissenschaften \\ Ontogenetic variation in cold tolerance plasticity in Drosophila: is the Bogert effect bogus? \\ --Manuscript Draft--}

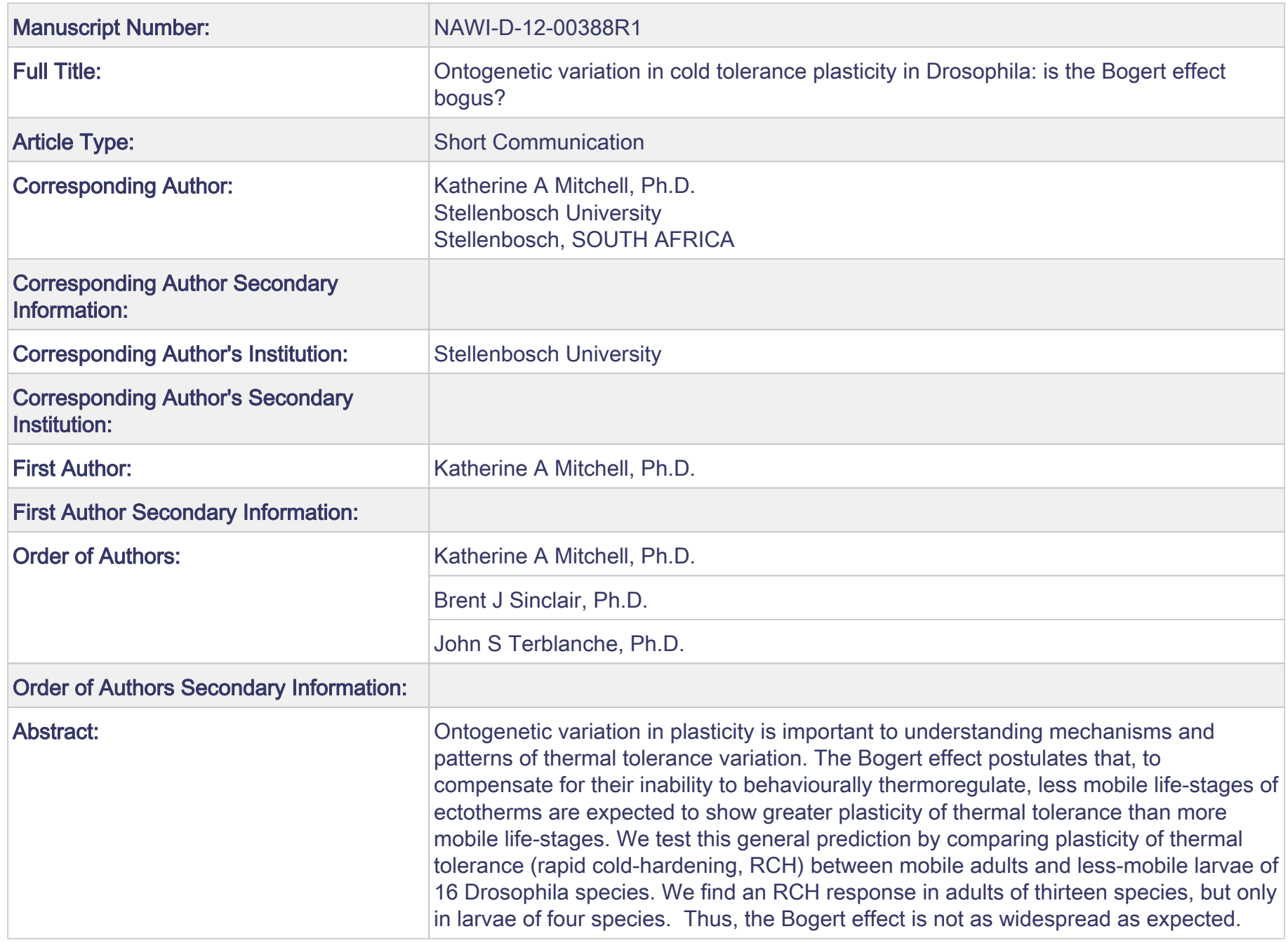


08-02-2013

Dear Dr. Thatje,

\section{$\underline{\text { RE: Manuscript re-submission (MS\# NAWI-D-12-00388) }}$}

Thank you for your email containing the responses from reviewers for our manuscript "Ontogenetic variation in cold tolerance plasticity in Drosophila: is the Bogert effect bogus?" by Mitchell, Sinclair and Terblanche for reconsideration of publication in Naturwissenschaften. We are pleased with the reviewer's general enthusiasm for the manuscript and are grateful for the opportunity to make improvements according to their input and resubmit to Naturwissenschaften.

We have carefully addressed the reviewer's comments and updated our manuscript accordingly. Below, we provide a response to each reviewer's comments and highlight the corresponding changes to the manuscript (responses provided in italics). The overarching theme from all reviewer's was their concern regarding the different methodology adopted to assess rapid cold hardening between the adult and larval life stages i.e. species-specific vs. standard pre-treatment for adults and larvae, respectively. We do openly acknowledge these issues in the manuscript (bearing in mind that we did not conduct these studies de novo, but are instead making use of published data) and we have accounted for this potential confounding factor by not using absolute levels of plasticity but rather the presence/absence of rapid cold hardening within each life stage. Perhaps we did not make it clear in the earlier version that this was the basis for all analyses and have clarified this in the revised manuscript. As the intention of this article was to refocus attention on the importance of the Bogert effect and encourage further investigation rather than intensely scrutinize it, we acknowledge that our study contains some caveats. Despite these caveats, we do feel that this is the best available dataset to assess the question in a systematic fashion across a range of species. We anticipate that the revived interest that should be generated by our paper will result in new data and aid to quickly advance the research in this field; this paper should therefore result in significant citations and be a benchmark assessment of this idea.

All authors agree to the alteration and resubmission of this manuscript which contains new results not currently in review or published elsewhere. The word count for the manuscript is 2406 words.

We hope that the following changes will make our manuscript suitable for publication in Naturwissenschaften and we look forward to hearing from you.

Kind regards,

Dr. Katherine Mitchell (corresponding author)

Stellenbosch University 
Reviewers' comments:

Reviewer \#1: Review of Naturwissenschaften ms NAWI-D-12-00388: Ontogenetic variation in cold tolerance plasticity in Drosophila: is the Bogert effect bogus? by Mitchell et al.

This short paper addresses the prediction that evolution favors phenotypic plasticity in cold tolerance traits in immobile organisms/life stages to compensate for the lack of regulation through behavioral means. The (cited) empirical tests of the prediction come from insects of sub-Antarctic origin, evolved in an extreme environment, which might not be representative for insects generally. Thus, to explore the generality of the phenomenon data from 16 species of Drosophila is investigated here.

The paper compares two published data-sets, one on adult rapid cold hardening $(\mathrm{RCH})$ ability and one 3rd instar larval RCH ability. The paper compared the rate of RCH ability among the two life stages and find that RCH is more wide spread in adults than in larvae, and thus do not find support for the prediction, as larvae are assumed able to behaviorally thermo-regulate to a lesser degree than adults. There are a number of challenges when using a collection of species in common garden experiments, e.g. that all species might be adapted to different environments originally, might be fed more or less optimal food or might be kept at more or less optimal food. There is no easy fix, but maybe this should be acknowledged and discussed briefly.

Response: The referee is correct. We acknowledge that maintaining flies in a common environment does remove some of the ecological relevance of the experiments, however, as the reviewer acknowledges, this is a "catch-22" situation. Without having them all reared under common conditions, readers could argue that differences in plasticity/tolerance or a lack thereof was simply a consequence of different thermal history or local environmental conditions. We haven't specifically referred to the potential of laboratory food being an issue but we feel that the statement 'lab adaptation' encompasses all of these elements.

The idea is good and the writing, analyses, and discussion are all clear and done appropriately. Still, I can't help thinking that the paper yield rather limited insight to this issue. The authors discuss reasons for the lack of support for their prediction: 1) methodological differences between the studies investigating adults and larvae, 2 ) evolutionary constraints (in the Drosophila genus) and/or 3) that the phenomenon is trait specific and do not apply to low temperature (as measured in the studies).

Response: We are grateful for the positive comments regarding the general structure and writing of the manuscript, however we respectfully disagree regarding the limited insight of our study. It is rather easy in hindsight to criticise the outcomes of this work, especially when the overall outcome is a lack of support for an intuitively appealing idea. The Bogert effect is an important theory that has received very little attention following its initial inception (Huey et al. 2003) and subsequent testing (Marais \& Chown 2008), and has certainly never been examined across such a broad cross-section of species. Our inability to find any evidence for the Bogert effect already highlights that there are certainly exceptions to this theory and will hopefully spur more systematic examination across different taxonomic groups and traits. Nevertheless, care is taken to discuss the potential limitations of the work so that others who carry this work foreward are suitably armed to tackle the question thoroughly. Thus, we believe that (since it is only the second explicit test of the Bogert Effect), the insight yielded by this paper is important, especially because the possible explanations we put forward lead to clear, testable hypotheses that will shed further light on the nature of the evolution of plasticity. Such hypotheses would not have been generated without this study. 
Already when reading the abstract the first time I noted down: "is this trait [RCH as measured here] relevant for both life stages of these Drosophila species?". It is a prerequisite for the whole idea that the trait in question (ability to rapidly cold harden to sub-zero cold shock) is under (equally strong) directional selection in both life stages. I am not convinced that this is so. To argue their case a number of traits should be investigated and compared. In line with this, I get the feeling that the ms suggests that "plasticity" in general have been measured for these species, while in reality "plasticity for $\mathrm{RCH}$ " have been measured. This ought to be clear in the writing, as the paper seems to take on a much broader generality than it can carry.

Response: We respectfully disagree with the reviewer on these points. It is well acknowledged that cold tolerance is a significant limiting factor for ectotherm distributions, particularly in Drosophila (e.g. Kimura 1988, Evolution 42, 1288-1297), and the fact that larvae have received little consideration with respect to this trait is quite an oversight. In general, larvae have been found to have greatly reduced thermal tolerance compared to other life stages, however this is true for both heat and cold tolerance (e.g. Krebs \& Loeschcke 1995 Biol. J. Linn. Soc. 56, 517-531; Jensen et al. 2007), providing no indication of differing selective pressures between upper and lower thermal tolerances. As the evidence for rapid cold hardening is determined relative to controls for each species and within each life stage, and because each species is then subjected to a stress which is 'tailored' to elicit a particular stress response i.e. the stress level of $90 \%$ lethality, we believe any potential confounding elements have been eliminated. The reduced survival in the larvae relative to adults for rapid cold hardening is a factor to be considered however, as stated above, this is a situation that occurs for both heat and cold tolerance traits so likely to be a realistic effect of this stress on the larval stage. We certainly agree that more traits should be examined for the Bogert effect to gain a better understanding of the situations where it may/may not apply (and we identify this in the third possible explanation for the discrepancy between our results and Marais \& Chown's, noted above), however this is outside the scope of our particular study.

It was certainly not our intention to convey a broader generality than plasticity within rapid cold hardening and have carefully edited the manuscript to reflect this. Thank you for bringing it to our attention. Note that because RCH is a form of plasticity, "plasticity for $R C H$ " is redundant.

Please check lines 118 and 120. It seems to me that something is mixed up or is unclear to me. Line 118 stated that only two species (borealis and mojavensis) do not show adult RCH, and in line 120 it states than pseudoobscura shows no RCH either. That makes it three species showing no RCH in adults (borealis, mojavensis and pseudoobscura)?

Response: The reviewer is correct, thank you for bringing this to our attention. As the reviewer points out, 13/16, rather than 14/16 species showed a RCH response in adults. We have modified the ms to reflect this, and updated the statistics, but this minor error does not alter the results of those analyses, nor does it have any impact on our conclusions.

\section{Reviewer \#3:}

To authors,

there are some minor points which deserve improvements or correction Bogert: it would be very nice to have more information: who was Bogert and what was the reference paper.

Response: Due to the short format of this article, we are limited by how much information we can provide and the historical context of who Bogert was is not altogether relevant for understanding the principle. However, at the first mention of the Bogert effect we have included the primary referenceHuey et al. 2003, which we feel should be sufficient for the curious reader. Huey et al. 2003 spend 
some time dealing with these issues in their paper, but we feel this would detract from the focused paper we are aiming for.

line 112:the values indicated, $-\mathbf{- 1 . 5}$ and -10 seem to be wrong: -1.6 and-10.7.

Response: We had provided general values to make the point, but we now see that this is confusing. We have now changed these to the actual values. Thank you for highlighting this.

Table 1 and line 118. I do not understand the way species are counted; for example, adults pseudobscura should be considered as a lack of response.

Response: This was an oversight that we have now corrected (please see the response to previous reviewers comments). Thank you for pointing this out.

References: The name of the authors are given in text, while there are numbers in the list: please choose!

Response: This was a formatting error that has now been corrected. Thank you for pointing this out to us.

Reviewer \#3:

Mitchell et al's current paper uses currently published data to compare, across species, levels of phenotypic plasticity in cold tolerance in larvae and adults. The intention of this paper is examine the hypotheses of the Boggert effect which proposes that less mobile life-stages of ectotherms should display greater levels of plasticity as they are unable to thermo-regulate as easily as their adult counterparts. The question is interesting and relevant particularly from an evolution of plasticity and climate change perspective and the paper is well written. The authors found most adult species were able to rapidly cold harden while most larvae could not. My biggest concern with this paper is the way in which plasticity is measured and compared across the different species and life stages. For adults specific treatments were used for each species, the treatments were to a certain degree chosen to induce a plastic response. In contrast a single set treatment was chosen for the larvae. Clearly this treatment was much more stressful as evidenced by much lower RCH viability estimates for the larvae (on average $12 \%$ vs $51 \%$ ) and I can't help wonder if different treatments were used would we see a different result? It is at least my opinion that it is much more difficult to prove an absence of plasticity or at least requires an extensive comparison across a number of treatments. We also get into the issue of how to compare relevant measures of plasticity across species and this is difficult. The authors have chosen the temperature at which $90 \%$ mortality occurred in basal flies (?) and then taken the \%survival of pre-treated - \% survival of basal. Using this method it becomes very difficult to know exactly what it is you are comparing and what it actually means ecologically. The authors do address these issues in the second paragraph of their discussion but I can't help wondering whether the data is sufficiently robust enough to test the proposed hypotheses?

Response: We appreciate the reviewer's compliments of our manuscript and acknowledge their hesitation regarding the estimation of plasticity between the life stages. We have outlined our position in the response to the previous reviewer and in the cover letter, and believe the non-parametric approach we adopted for comparisons is more conservative than using the raw values which may be affected to a larger degree by the methodology. Please see our response to reviewer one for more details. We disagree that this makes it difficult to know what we are testing ecologically; in fact, it seems more relevant to us as, if the thermal tolerance of each species is determined somewhat by the environment they are adapted to, then by using specific stressors for each species and life stage, we are reflecting conditions that a particular organism and life stage would find stressful. 
Line 36-39 I don't think an overlooked failing of the ramping debate is a focus on adults rather than larvae? But I don't think this is what the authors mean.

Response: We thank the reviewer for drawing this oversight to our attention. Indeed, we do not believe the ramping debate needs the inclusion of larval data at this stage, but rather the focus on adult resistance for thermal tolerance in general is a failing of studies seeking biogeographic correlates of distribution as it seems more likely that the more susceptible larval life stage may limit species distributions more so than adults. We have now clarified our position in the manuscript.

The authors should mention in their introduction that the effect of $\mathrm{RCH}$ is not constant at either the adult stage (Kelty and Lee, 2001) or in the larval stage (Jensen et al - already cited).

Response: This is an important point that the reviewer has made and we have now addressed this by the inclusion of "This is not altogether surprising since cold tolerance plasticity varies considerably both within and between life stages (e.g. Kelty \& Lee 2001; Jensen et al. 2007)." at lines 55-57 in the manuscript. We thank the reviewer for bringing this to our attention.

Line 82: they choose third instar lavae - an ealier report on D. melanogaster (Jensen) has shown that this stage does not respond to $\mathrm{RCH}$, while an earlier stage does!?

Response: The Jensen et al. paper focuses only on D. melanogaster, whereas we used a dataset encompassing many species, and in which some wandering larvae do display RCH. Wandering larvae are easy to work with (hence their use in the Strachan et al. paper from which we obtained the data). Jensen et al. (2007) showed significant variation in RCH between and within life stages of $\mathrm{D}$. melanogaster, so it would be possible to use that dataset to argue against any life stage we chose.

Line 99-101 I am confused did you do your own statistical tests? Or just base conclusions on previous published data? Line 106-108 Authors need to be a little more explicit on their methods here, were branch lengths genetic distances?

Response: We thank the reviewer for highlighting these potential points of confusion. We did conduct our own statistical tests on the proportion of species within each life stage that showed RCH, however we used the analysis from the original papers to determine these proportions. We have now clarified this in lines 94-96 by stating "We compared the proportion of each life stage showing RCH between life stages across the 16 species using a two-proportion z-test (Statistica, StatSoft Inc. 2011). Significant RCH responses were determined based on statistical tests in the original papers (Nyamukondiwa et al. 2011; Strachan et al. 2011)."

The phylogenetic tree was constructed using branch lengths estimated from genetic sequence data. This has been clarified in the manuscript at lines 99-101 by inclusion of the following statement "We used a pruned phylogenetic tree obtained from Nyamukondiwa et al. (2011; branch lengths calculated from nucleotide sequences) to identify phylogenetic constraints on the ability of adults or larvae to mount an $\mathrm{RCH}$ response."

Line 152-154 There is some data to suggest inbreeding doesn't affect plastic responses in Drosophila species Kristensen et al 2011, they need to qualify this statement.

Response: We thank the reviewers for drawing this paper to our attention and have now included ths statement "This may have been unnecessary, however, as inbreeding depression that is likely to occur in the laboratory environment has no significant influence on plastic responses for cold tolerance in multiple Drosophila species (Kristensen et al. 2011)." in lines 144-146 of the manuscript. 
Line 155-156 How would looking at discrete traits and within a phylogenetic context alleviate this problem?

Response: We have addressed this concern in our response to the previous reviewer but will briefly summarise here. By using conservative estimates of plasticity estimated within each species and life stage plus controlling for phylogeny, we should be able to remove any confounding elements of the different methodologies used and, if we find a pattern, know it must be robust.

Line 159-162 I would be careful to interpret phylogenetic signal as phylogenetic constraint

Response: We have used the original author's interpretation of their results in our statement here but do agree that this is a relevant conclusion, based not only on the phylogenetic signal present but also the patterns seen in tolerance and plasticity between the different species.

Line 163-165 I am not sure what the authors mean here

We have now clarified sentence by replacing with the following; "The absence of a phylogenetic effect in this study may result from our analysis of the presence/absence of plasticity rather than an absence of signal in these traits in general, as the phylogenetic signal may be more subtle than such broad estimates can detect."

Line 170 this sentence is confusing are the authors trying to say there are likely to be greater differences in the thermal environment of larvae vs adults?

Response: We now understand the confusion that this sentence may cause and have reworded this to "The potential thermal environment of adult and larval Drosophila likely differs more than between the larvae and (flightless) adults of $P$. dreuxi (Klok and Chown 2001; Marais and Chown 2008) due to the limited dispersal potential and, therefore, close proximity of $P$. dreuxi adults to larval habitats." at lines 160-163. We thank you for pointing out these issues and hope to have clarified this in the revised manuscript.

\section{Reviewer \#4:}

In this manuscript the authors investigate ontogenetic variation in cold tolerance plasticity in multiple Drosophila species. They specifically test the hypothesis that less mobile life stages show greater plasticity than do more mobile life stages. To test this hypothesis they investigate 16 species of Drosophila. In contrast to the expectations they find that adults are more plastic than larvae.

The idea tested is important in evolutionary physiology and the experimental set up is straight forward and described clearly. The same goes for the result section and the conclusions drawn are generally supported by the data. In summery I believe that the ms will become a nice contribution to the literature.

One criticism however is that the species specific method used to determine pre-treatment temperatures for adults, which is elegant, should have been used for larvae as well to fully justify the comparison between plasticity in the two life stages.

Response: Thank you for your positive comments. Again, the issue of methodology between the adult and larvae studies has been raised by the other reviewers - as we have explained, we were constrained by the availability of the pre-existing datasets. Please see our earlier responses to these concerns. 
Further the ecological relevance of the study is debatable (as mentioned by the authors) since some of the species are never exposed to the thermal regimes investigated in the laboratory in their natural habitat and because laboratory stock are mainly used in this study.

However despite this criticism the result represents a valuable and novel contribution to the literature and paves the way for further studies potentially performed under more ecological relevant conditions. In relation to the ecological relevance further information about thermal microclimates of the investigated species in their natural habitats would be relevant to obtain in further studies. Those data could be useful in dictating the relevant acclimation regimes. Also I suggest that performing experiments testing the Bogert effect in the field are necessary for a better understanding of the evolutionary significance of thermal plasticity.

Response: Ecological relevance is also a concern that we have addressed previously-please see our earlier comments. We are hesitant to draw conclusions about natural populations from our study due to the use of lab stocks but this is definitely something that needs to be addressed in future using natural populations. We don't have the data about the thermal environment for each species to make such statements (and in any case, because some of these stocks have been in the lab for decades, they would be difficult statements to justify). However, plasticity has clearly been maintained in the lab colonies, and the adults are still more mobile than the larvae, so as a test of the Bogert effect, we believe our approach to be robust. We agree wholeheartedly with the reviewer on the need for natural and field estimates. Such concerns have been echoed here and in our previous work (e.g. see discussion in Terblanche et al. 2011).

Minor issues:

Line 36: 'The methology used in such studies...' instead of 'The methology of such studies..'? Response: This has now been changed. Thank you for drawing this to our attention.

Line 134: substitute 'significant' with 'likely'

Response: Now changed, thank you.

Line 159-162 I would be careful to interpret phylogenetic signal as phylogenetic constraint 163-165 I am not sure what the authors mean here Line 170 this sentence is confusing are the authors trying to say there are likely to be greater differences in the thermal environment of larvae vs adults?

Response: We apologise for the confusion and have now clarified these statements. We have replaced the word "constraint" with "signal" in the sentence "Both of the studies from which we extracted data found significant phylogenetic signal in the plasticity of cold tolerance as well as a negative correlation between RCH and basal resistance (Nyamukondiwa et al. 2011; Strachan et al. 2011)." at lines 148-151. 
1 Ontogenetic variation in cold tolerance plasticity in Drosophila: is the Bogert

\section{2 effect bogus?}

3

4 Katherine A. Mitchell ${ }^{1}$, Brent J. Sinclair ${ }^{2}$ \& John S. Terblanche ${ }^{1}$

$5{ }^{1}$ Department of Conservation Ecology \& Entomology, Faculty of AgriSciences,

6 Stellenbosch University, Private Bag X1, Matieland 7602, South Africa

$7 \quad{ }^{2}$ Department of Biology, University of Western Ontario, London, ON, Canada N6A

$8 \quad 5 B 7$

$9 *$ *orresponding author e-mail: kmitchell@sun.ac.za

10

11

12

13 Short Title: Testing the Bogert effect in Drosophila 


\section{Abstract:}

16 Ontogenetic variation in plasticity is important to understanding mechanisms and

17 patterns of thermal tolerance variation. The Bogert effect postulates that, to

18 compensate for their inability to behaviourally thermoregulate, less mobile life-stages

19 of ectotherms are expected to show greater plasticity of thermal tolerance than more

20 mobile life-stages. We test this general prediction by comparing plasticity of thermal

21 tolerance (rapid cold-hardening, $\mathrm{RCH}$ ) between mobile adults and less-mobile larvae

22 of 16 Drosophila species. We find an $\mathrm{RCH}$ response in adults of thirteen species, but

23 only in larvae of four species. Thus, the Bogert effect is not as widespread as

24 expected.

25

26 Keywords: behavioural thermoregulation, phenotypic plasticity, climate

27 variability, development

28 


\section{Introduction}

30 The thermal tolerances and geographic distributions of ectotherms are linked, and

31 understanding thermal limits can shed light on responses to climate change (e.g.

32 Angilletta 2009). The methodology used in such studies has been debated (e.g.

33 Rezende and Santos 2012; Terblanche et al. 2011), and does not incorporate

34 adaptation potential into predictions (Hoffmann \& Sgrò 2011). An overlooked failing

35 of biogeographic association studies is the emphasis on adult-stage thermal tolerance.

36 This adult bias is particularly problematic for holometabolous insects, whose larval

37 stages may have limited mobility among thermal microhabitats and whose thermal

38 limits can differ significantly from the adult stages (Bowler \& Terblanche 2008).

39 Ontogenetic differences in thermal limits may confound understanding of species'

40 distributions and climate change responses. This is particularly true if thermal

41 tolerances of the more sedentary, and potentially more susceptible, life-stages set

42 distributional limits.

43 Behavioural thermoregulation allows ectotherms to avoid extreme conditions,

44 so more mobile ectotherms should experience reduced selection for physiological

45 plasticity (the Bogert effect; Huey et al. 2003). Marais and Chown (2008) suggested

46 that the Bogert effect applies among life stages of holometabolous insects (where

47 adults may be more mobile than larvae). Chill coma recovery time (CCR) was more

48 plastic in larvae than adults of the sub-Antarctic kelp fly (Paractora dreuxi; Marais

49 and Chown 2008), supporting the Bogert effect, although larvae were less tolerant of

50 extreme temperatures than adults. Evidence for the Bogert effect from other

51 arthropods is sparse. Larvae, but not adults, of Belgica antarctica are capable of rapid

52 cold-hardening $(\mathrm{RCH})$, an acute phenotypic plasticity of thermal tolerance (Lee et al.

53 2006). Another study of $P$. dreuxi found no support for the Bogert effect when 
54 examining survival following cold shock rather than CCR (Marais et al. 2009),

55 indicating that the Bogert effect applies to only some traits. This is not altogether

56 surprising since cold tolerance plasticity varies considerably both within and between

57 life stages (e.g. Kelty \& Lee 2001; Jensen et al. 2007). These results of previous

58 studies of the Bogert effect are difficult to compare due to variation in the traits

59 measured, and can shed little light on overall evolutionary patterns as each examines

60 only a single species.

61 To investigate the Bogert effect in a broader, evolutionary context, we directly

62 compare $\mathrm{RCH}$ in the same suite of species at the larval and adult life-stages. We

63 utilized two pre-existing datasets containing estimates of the magnitude of $\mathrm{RCH}$

64 responses for larvae (Strachan et al. 2011) and adults (Nyamukondiwa et al. 2011) of

6516 Drosophila species. We predicted that if the Bogert effect drives the evolution of

66 phenotypic plasticity of thermal tolerance, plastic responses that improve cold

67 tolerance should be more prevalent in the larvae, the least mobile life-stage.

68

69

Material \& Methods 
10 individuals were cooled from $10^{\circ} \mathrm{C}$ to $0^{\circ} \mathrm{C}$ at $0.5^{\circ} \mathrm{C} / \mathrm{min}$, then at $0.1^{\circ} \mathrm{C} / \mathrm{min}$ to a

80 range of predetermined temperatures, where they were held for $1 \mathrm{~h}$. Larvae were

81 rewarmed to $10^{\circ} \mathrm{C}$ at $1^{\circ} \mathrm{C} / \mathrm{min}$ and survival scored as successful adult eclosion. A

82 hardening response was induced by exposing larvae for $1 \mathrm{~h}$ at $4^{\circ} \mathrm{C}$ followed by $1 \mathrm{~h}$ at

$83 \mathrm{LLT}_{90}$, and survival was compared to that of controls that had not received the RCH

84 pre-treatment.

85 A similar method was used for $\mathrm{LLT}_{90}$ in adults (Nyamukondiwa et al. 2011),

86 except that survival was estimated following acute transfer to the test temperatures

87 rather than the temperature ramp. Hardening was induced by exposing groups of 4-6

88 day old flies to a temperature $10^{\circ} \mathrm{C}$ above the estimated $\mathrm{LLT}_{90}$ for $2 \mathrm{~h}$ before exposure

89 to the $\operatorname{LLT}_{90}$, resulting in a unique combination of temperatures for each species.

90 Flies that exhibited coordinated movement were considered alive.

91 To quantify the plasticity exhibited by each life stage, we calculated the

92 percentage difference in survival (hereafter referred to as $\mathrm{RCH}$ effect) as

93 survival $_{\text {pretreated }}-$ survival $_{\text {control, }}$, (negative values indicate that the $\mathrm{RCH}$ pre-treatment

94 reduced cold tolerance) from the datasets. We compared the proportion of each life

95 stage showing $\mathrm{RCH}$ between life stages across the 16 species using a two-proportion

96 z-test (Statistica, StatSoft Inc. 2011). Significant RCH responses were determined

97 based on statistical tests in the original papers (Nyamukondiwa et al. 2011; Strachan

98 et al. 2011).

99 We used a pruned phylogenetic tree obtained from Nyamukondiwa et al.

100 (2011; branch lengths calculated from nucleotide sequences) to identify phylogenetic

101 constraints on the ability of adults or larvae to mount an $\mathrm{RCH}$ response. We

102 conducted a discrete character maximum likelihood model comparison using Discrete 
103 in BayesTraits (Pagel and Meade 2008), and compared the log-likelihood ratio

104 between models dependent and independent of phylogeny.

105

106

107

108

109

110

111

112

113

114

115

116

117

118

$\left.119 \chi^{2}=3.66, \mathrm{df}=2, \mathrm{p}=0.15\right)$. compared to the more-mobile adults. This suggests that the Bogert effect is not

124 broadly evident in this genus, and that the clear Bogert effect in Paractora dreuxi cold 125 tolerance (Marais and Chown 2008) may be the exception rather than the rule. Three 126 potential explanations for this outcome appear most likely. 
First, the differences in the methods used to induce $\mathrm{RCH}$ responses in the two

128 studies might have biased the detection of plasticity towards adults. The species-

129 specific determination of adult pre-treatment temperatures likely maximised the

130 likelihood of detecting plasticity compared to the uniform $\mathrm{RCH}$ pre-treatment used for

131 larvae. This may be particularly true if the uniform pre-treatment temperature injured

132 some larvae. For example, larvae of $D$. simulans had reduced cold hardiness after the

$133 \mathrm{RCH}$ pre-treatment, but $\mathrm{RCH}$ pre-treatment increased adult survival by $75 \%$.

134 However, the pre-treatment temperature $\left(4\right.$ and $5^{\circ} \mathrm{C}$ for larvae and adults) and the

135 estimates of $\operatorname{LLT}_{90}\left(-3.7\right.$ and $\left.-5^{\circ} \mathrm{C}\right)$ were similar for adults and larvae. Our results are

136 also consistent with other studies of $D$. melanogaster, which report little to no RCH in

137 larvae but significant RCH in adults (Jensen et al. 2007).

138 The use of laboratory stocks prevent us from drawing specific conclusions

139 about the ecological relevance of the results for these species in the field. However,

140 we would expect laboratory adaptation to alter the magnitude of plasticity rather than

141 the presence or absence of a hardening response, and to act on both adults and larvae

142 in equal measure. For this reason we analysed the data conservatively, employing

143 discrete traits and accounting for potential (but non-significant) phylogenetic signal.

144 This may have been unnecessary, however, as inbreeding depression that is likely to

145 occur in the laboratory environment has no significant influence on plastic responses

146 for cold tolerance in multiple Drosophila species (Kristensen et al. 2011).

147 Second, our results may reflect evolutionary constraints - or even selection

148 against the Bogert effect - acting across the Drosophila genus. Both of the studies

149 from which we extracted data found significant phylogenetic signal in the plasticity of

150 cold tolerance as well as a negative correlation between $\mathrm{RCH}$ and basal resistance

151 (Nyamukondiwa et al. 2011; Strachan et al. 2011). Thus, RCH is limited by 
152 evolutionary history and as a trade-off with general cold tolerance. The absence of a 153 phylogenetic effect in this study may result from our analysis of the presence/absence

154 of plasticity rather than an absence of signal in these traits in general, as the

155 phylogenetic signal may be more subtle than such broad estimates can detect. There 156 are also ecological reasons why Drosophila may not show a Bogert effect. Larvae 157 avoid thermal extremes, possibly aided by thermally-selective choice of oviposition 158 sites (Dillon et al. 2009), although both adults and larvae of D. melanogaster do 159 experience thermal stress in the field (Feder et al. 2000; Roberts and Feder 1999).

160 The potential thermal environment of adult and larval Drosophila likely differs more 161 than between the larvae and (flightless) adults of P. dreuxi (Klok and Chown 2001; 162 Marais and Chown 2008) due to the limited dispersal potential and, therefore, close 163 proximity of $P$. dreuxi adults to larval habitats.

164 Third, the Bogert effect may be trait-specific, and not apply to low

165 temperature mortality (as we examined). The Bogert effect is observed in chill coma 166 recovery (Marais and Chown 2008), but not low temperature mortality of $P$. dreuxi 167 (Marais et al. 2009). Movement at low temperatures is essential for avoiding low 168 temperature mortality and predation, and is therefore a clear target for selection 169 associated with the ability to thermoregulate (as is proposed for the Bogert effect).

170 Thus, further work is needed to disentangle the traits and life stages where

171 behavioural mobility or innate thermal tolerance is the target of natural selection. We 172 suggest that thermal plasticity be explored across other arthropod groups and 173 additional life stages and the broad range of thermal tolerance traits that can be 174 measured in insects (Terblanche et al. 2011) to better understand the nature and limits 175 of the Bogert effect in ectotherms. 
178 JST and KAM are supported by FruitGro Science and the National Research

179 Foundation. BJS is supported by a Discovery Grant from the Natural Sciences and

180 Engineering Research Council of Canada.

181

182

183

184

185

186

\section{Conflict of interest}

The authors declare no conflict of interest.

\section{References}

Angilletta, M.J. (2009). Thermal adaptation: a theoretical and empirical synthesis. Oxford University Press, Oxford.

Bowler, K. \& Terblanche, J.S. (2008). Insect thermal tolerance: what is the role of ontogeny, ageing and senescence? Biol. Rev. 83, 339-355.

Dillon, M.E., Wang, G., Garrity P.A. \& Huey, R.B. (2009) Thermal preference in Drosophila. J. Therm. Biol. 34, 109-119.

Feder, M. E., Roberts, S. P. and Bordelon, A. C. (2000). Molecular thermal telemetry of free-ranging adult Drosophila melanogaster. Oecologia 123, 460-465.

Hoffmann, A.A. \& Sgrò , C.M. (2011). Climate change and evolutionary adaptation. Nature 470, 479-485.

Huey, R.B., Hertz, P.E. \& Sinervo, B. (2003) Behavioral drive versus behavioural inertia in evolution: a null model approach. Am. Nat. 161, 357-366.

Jensen, D., Overgaard, J. \& Sørensen, J. (2007) The influence of developmental stage on cold shock resistance and ability to cold-harden in Drosophila melanogaster. J. Insect Physiol. 53, 179-186. 
201 Kelty, J.D. \& Lee Jr., R.E. (2001) Induction of rapid cold hardening by cooling at ecologically relevant rates in Drosophila melanogaster. J. Insect. Physiol. 45, 719-726.

204

Klok, C.J. \& Chown, S.L. (2001) Critical thermal limits, temperature tolerance and water balance of a sub-Antarctic kelp fly, Paractora dreuxi (Diptera: Helcomyzidae). J. Insect Physiol. 47, 95-109.

Kristensen, T.N., Loeschcke, V., Bilde, T., Hoffmann, A.A., Sgrò , C., Noreikiené, K., Ondrésik, M., \& Bechsgaard, J.S. (2011) No inbreeding depression for low temperature developmental acclimation across multiple Drosophila species. Evolution 65, 3195-3201.

211 Lee, R.E., Elnitsky, M.A., Rinehart, J.P., Hayward, S.A., Sandro, L.H. \& Denlinger, D.L. (2006) Rapid cold-hardening increases the freezing tolerance of the Antarctic midge Belgica antarctica. J. Exp. Biol. 209, 399-406. < http://www.evolution.rdg.ac.uk/BayesTraits.html >

224 Rezende, E.L. \& Santos, M. (2012) Comment on 'Ecologically relevant measure of 225 tolerance to potentially lethal temperatures'. J. Exp. Biol. 215, 702-703. 
226 Roberts, S. P. and Feder, M. E. (1999). Natural hyperthermia and expression of the 227 heat shock protein Hsp70 affect developmental abnormalities in Drosophila 228 melanogaster. Oecologia 121, 323-329.

229 StatSoft, Inc. (2011). Statistica (data analysis software system), version 10.

$230 \quad$ www.statsoft.com

231 Strachan, L.A., Tarnowski-Garner, H.E., Marshall, K.E. \& Sinclair, B.J. (2011) The 232 evolution of cold tolerance in Drosophila larvae. Physiol. Biochem. Zool. 84, $233 \quad 43-53$.

234 Terblanche, J.S., Hoffmann, A.A., Mitchell, K.A., Rako, L., le Roux, P.C. \& Chown, 235 S.L. (2011) Ecologically relevant measures of tolerance to potentially lethal 236 temperatures J. Exp. Biol. 214, 3713-3725. 
Table 1: Summary of rapid cold hardening in larvae and adults of Drosophila species from Strachan et al. (2011) and Nyamukondiwa et al. (2011) (adults). LLT $_{90}=$ species-specific low temperature that causes $90 \%$ mortality. $\mathrm{RCH}$ effect is the percentage improved survival after pre-treatment. Pre-exposure temperature for adults was $10^{\circ} \mathrm{C}$ above species-specific $\mathrm{LLT}_{90}$. Pre-exposure temperature for larvae was set at $4^{\circ} \mathrm{C}$ across species.
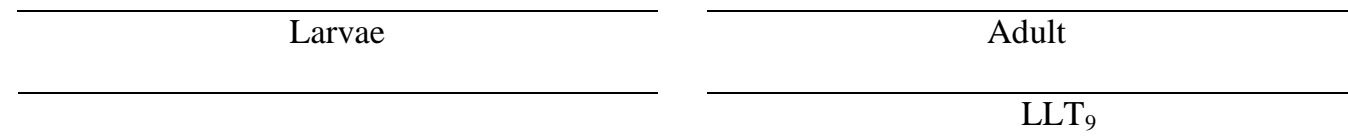

Pre-exposure

$\mathrm{LLT}_{90} \quad \mathrm{RCH}$ effect

Temperature $\left({ }^{\circ} \mathrm{C}\right)$

(\% survival)

Pre-exposure

$\mathrm{RCH}$ effect

Temperature $\left({ }^{\circ} \mathrm{C}\right)$

$\left({ }^{\circ} \mathrm{C}\right)$

(\% survival)

Species

\begin{tabular}{|c|c|c|c|c|c|c|}
\hline D. ananassae & 4 & -3.8 & 29 & 7 & -3 & 80 \\
\hline D. auraria & 4 & -5.4 & 10 & 2 & -8 & 45 \\
\hline D. borealis & 4 & -9.5 & 44 & -3 & -13 & 10 \\
\hline D. erecta & 4 & -3.4 & -3 & 5 & -5 & 90 \\
\hline D. hydei & 4 & -6.1 & 20 & 3 & -7 & 40 \\
\hline D. immigrans & 4 & -5.2 & 10 & 6 & -4 & 60 \\
\hline D. melanogaster & 4 & -4.7 & 10 & 5 & -5 & 80 \\
\hline D. mojavensis & 4 & -6.8 & -7 & 4 & -6 & 10 \\
\hline D. persimilis & 4 & -9.6 & 47 & -2 & -12 & 15 \\
\hline D. pseudoobscura & 4 & -10.7 & 0 & -2 & -12 & 0 \\
\hline D. sechellia & 4 & -1.6 & 7 & 6 & -4 & 65 \\
\hline D. simulans & 4 & -3.7 & -17 & 5 & -5 & 75 \\
\hline D. takahashii & 4 & -4.1 & 6 & 6 & -4 & 85 \\
\hline D. virilis & 4 & -10.7 & 14 & -1 & -11 & 40 \\
\hline D. willistoni & 4 & -3.8 & 21 & 6 & -4 & 50 \\
\hline D. yakuba & 4 & -4.3 & 7 & 6 & -4 & 80 \\
\hline
\end{tabular}

